\title{
Entamoeba histolytica Infection in Female Sex Workers: A Matched Case-Control Study in Durango, Mexico
}

\author{
Cosme Alvarado-Esquivelel, d, Luis Francisco Sanchez-Anguiano ${ }^{\mathrm{b}}$, Jesus Hernandez-Tinoco ${ }^{\mathrm{b}}$, \\ Sergio Estrada-Martinez ${ }^{\mathrm{b}}$, Alma Rosa Perez-Alamos ${ }^{\mathrm{b}}$, Agar Ramos-Nevarez ${ }^{\mathrm{c}}$, \\ Sandra Margarita Cerrillo-Soto ${ }^{\mathrm{c}}$, Carlos Alberto Guido-Arreola ${ }^{\mathrm{c}}$
}

\begin{abstract}
Background: Infection with Entamoeba histolytica (E. histolytica) can be potentially transmitted by sexual contact. The seroepidemiology of $E$. histolytica in female sex workers has not been studied. The aim of the study was to determine whether E. histolytica is associated with the occupation of female sex work. In addition, the correlates of E. histolytica seroprevalence in female sex workers were also investigated.
\end{abstract}

Methods: We performed an age- and gender-matched case-control study of 187 female sex workers and 374 women without sex work. Cases and controls were tested for the presence of E. histolytica IgG antibodies using a commercially available enzyme-linked immunoassay. Seroprevalence association with the characteristics of female sex workers was determined by bivariate analysis.

Results: Anti-E. histolytica IgG antibodies were found in five $(2.7 \%)$ of 187 female sex workers and in $16(4.3 \%)$ of 374 controls (odds ratios $(\mathrm{OR})=0.61 ; 95 \%$ confidence intervals $(\mathrm{CI}): 0.22-1.70 ; \mathrm{P}=0.34)$. Mean optical density of the immunoassay in seropositive cases and controls was $1.35 \pm 0.93$ and $0.73 \pm 0.45$, respectively $(\mathrm{P}=0.05)$. Seroprevalence of $E$. histolytica infection did not vary significantly with age, education, socioeconomic level, or health status of sex workers. Seropositivity to $E$. histolytica did not correlate with work characteristics such as duration in the occupation, condom use, type of sex, or a history of sexually transmitted diseases, or with behavioral variables such as washing hands before eating, or consumption of untreated water.

Conclusions: Results indicate that female sex workers do not have an increased risk for E. histolytica infection in Durango City, Mexico.

Manuscript accepted for publication May 05, 2017

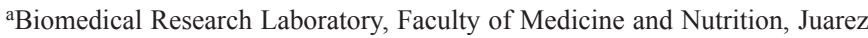
University of Durango State, Avenida Universidad S/N, 34000 Durango, Mexico

bInstitute for Scientific Research "Dr. Roberto Rivera-Damm", Juarez University of Durango State, Avenida Universidad S/N, 34000 Durango, Mexico ${ }^{\mathrm{c} C l i n i c a}$ de Medicina Familiar, Instituto de Seguridad y Servicios Sociales de los Trabajadores del Estado, Predio Canoas S/N, 34079 Durango, Mexico ${ }^{\mathrm{d} C}$ Corresponding Author: Cosme Alvarado-Esquivel, Laboratorio de Investigacion Biomedica, Facultad de Medicina y Nutricion, Avenida Universidad S/N, 34000 Durango, Dgo, Mexico. Email: alvaradocosme@yahoo.com

doi: https://doi.org/10.14740/jocmr3065w
Further studies to determine the risk of infection with $E$. histolytica by sexual contact should be conducted.

Keywords: Entamoeba histolytica; Case-control study; Epidemiology; Female sex workers; Risk factors; Mexico

\section{Introduction}

Entamoeba histolytica (E. histolytica) is a protozoa parasite causing infections in humans all over the world $[1,2]$, and represents a major health problem in developing countries [3]. Most infections with E. histolytica are asymptomatic [1]; however, some individuals may develop amebic colitis and life-threatening abscesses [4, 5]. E. histolytica is the second leading cause of death due to a protozoan parasite [6]. Transmission of $E$. histolytica usually occurs by fecal excretion of cysts followed by oral ingestion of contaminated drinking water or food [7]. However, infection with E. histolytica can also be sexually transmitted [8,9]. Several studies have shown an increased seroprevalence of $E$. histolytica infection in men who have sex with men (MSM) [10-12]. In addition, the seroprevalence of infection with $E$. histolytica is higher in HIV-positive MSM than HIV-negative MSM [13-15]. Sexually active HIV-positive MSM coinfected with $E$. histolytica was at greater risk of developing amebic disease caused by $E$. histolytica than HIV-negative MSM and the general population [16]. Infection with E. histolytica can also be transmitted by heterosexual activity and female homosexual activity [7]. In a Japanese study, researchers observed an increase in the annual rate of anti-E. histolytica seropositivity in female outpatients at a sexually transmitted disease clinic in Tokyo [17]. Penile amebiasis has been reported in men who practiced unprotected anal intercourse [18]. Very few studies about the link between infection with E. histolytica and sexual activity in women exist. In an epidemiological study of sexual contacts of a female with an amebic liver abscess, researchers uncover a cluster of seven cases of amebiasis in whom oral-anal sex was common and the five female individuals were bisexual or homosexual [7]. In addition, infection with E. histolytica by the fecal-oral route among heterosexual couples might occur [19]. We hypothesize that the occupation of female sexual worker represents a risk for infection with E. histolytica. To 
the best of our knowledge, the association between this occupation and infection with $E$. histolytica has not been studied. Female sex workers are of epidemiological concern because of their potential role for dissemination of sexually transmitted infections. Therefore, we sought to determine the association between $E$. histolytica seropositivity and the occupation of female sex worker in Durango City, Mexico. In addition, E. histolytica seropositivity association with the socio-demographic and behavioral characteristics of the female sex workers studied was also investigated.

\section{Materials and Methods}

\section{Study design and women studied}

We performed an age- and gender-matched case-control study to determine the association of E. histolytica infection with the occupation of female sex worker in Durango City, Mexico from May 2016 to March 2017.

\section{Female sex workers}

One hundred and eighty-seven female sex workers were enrolled in the study. Inclusion criteria for the cases were: 1) current working as female sex workers for at least 1 year; 2) registered in the Clinics for Sanitary Inspection of the Municipal Government; 3) aged 18 years and older, and 4) who accepted to participate in the study. The mean age of the female sex workers was $33.31 \pm 10.24$ years (range: 18 - 67 years).

\section{Control women}

Three hundred and seventy-four control women with occupations other than sex work matched with female sex workers by age and gender were included in the study. Control women were randomly selected from the general population of the same Durango City. The mean age in controls was $33.27 \pm$ 10.21 (range: 18 - 67 years) and comparable with that in sex workers $(\mathrm{P}=0.96)$.

\section{Socio-demographic, clinical, work and behavioral charac- teristics of female sex workers}

Socio-demographic, work, clinical and behavioral characteristics of the female sex workers were obtained through an interviewer-administered standardized questionnaire. Socio-demographic items included age, birthplace, residence, educational level, and socioeconomic status. Clinical data in the female sex workers included health status (healthy or ill), presence or history of lymphadenopathy, frequent abdominal pain, headache or hepatitis. Work characteristics recorded in female sex workers were: duration in the occupation, condom use, condom breakage, practice of oral or anal sex, mean number of clients per week, history of injuries during sex work, and history of sexually transmitted diseases. Behavioral data included washing hands before eating, consumption of unpasteurized milk or untreated water, consumption of unwashed raw vegetables or fruits, frequency of eating away from home (in restaurants or fast food outlets), contact with soil (gardening or agriculture), and foreign travel.

\section{Detection of anti-E. histolytica IgG antibodies}

A blood sample $(5 \mathrm{~mL})$ was collected from each participant. Serum samples were obtained by centrifugation of blood, and kept frozen at $-20{ }^{\circ} \mathrm{C}$ until analyzed. Anti-E. histolytica $\mathrm{IgG}$ antibodies were detected in serum samples by a commercially available enzyme immunoassay "E. histolytica IgG (Amebiasis) ELISA" kit (Diagnostic Automation Inc., Calabasas, CA, USA). According to the kit's insert, this enzyme immunoassay has a sensitivity of $92 \%$ and a specificity of $100 \%$. All assays were performed following the instructions of the manufacturer. Positive and negative controls were run in each assay. Positive sera of participants were tested twice, and only repeatedly reactive sera were considered positive for anti-E. histolytica antibodies.

\section{Statistical analysis}

Data were analyzed with the software SPSS 15.0 (SPSS Inc., Chicago, IL, USA). For calculation of the sample size, we used a $95 \%$ confidence level, a power of $80 \%$, a 1:2 proportion of cases and controls, a reference seroprevalence of $28.2 \%$ [20] as the expected frequency of exposure in controls, and an odds ratio (OR) of 1.8. The result of the sample size calculation was 151 cases and 301 controls. We used the Student's $t$-test to compare the age in cases and controls. Comparison of optical densities of ELISA in cases and controls was performed with the Student's $t$-test. We determined the association between $E$. histolytica seropositivity and the characteristics of the sex workers with the two-tailed Fisher's exact test (because of small values). ORs and 95\% confidence intervals (CIs) were obtained, and a $\mathrm{P}$ value $<0.05$ was considered statistically significant.

\section{Ethical aspects}

The aims and procedures of this case-control study were explained to all participants, and a written informed consent was obtained from all of them. This study was approved by the Ethical Committee of the Institute for Security and Social Services of the State Workers in Durango City.

\section{Results}

Anti-E. histolytica IgG antibodies were found in five $(2.7 \%)$ of 187 female sex workers and in $16(4.3 \%)$ of 374 controls. There was no difference in the seroprevalences between cases and controls $(\mathrm{OR}=0.61 ; 95 \% \mathrm{CI}: 0.22-1.70 ; \mathrm{P}=0.34)$. Mean 
Table 1. Socio-Demographic Characteristics of Female Sex Workers and Seroprevalence of E. histolytica Infection

\begin{tabular}{|c|c|c|}
\hline Characteristic & No. of subjects & No. of positive cases \\
\hline \multicolumn{3}{|l|}{ Age groups (years) } \\
\hline 30 or less & 84 & 1 \\
\hline $31-50$ & 87 & 3 \\
\hline$>50$ & 16 & 1 \\
\hline \multicolumn{3}{|l|}{ Birth place } \\
\hline Durango State & 152 & 4 \\
\hline Other Mexican State & 35 & 1 \\
\hline \multicolumn{3}{|l|}{ Residence place } \\
\hline Durango State & 185 & 5 \\
\hline Other Mexican State & 2 & 0 \\
\hline \multicolumn{3}{|l|}{ Residence area } \\
\hline Urban & 172 & 5 \\
\hline Suburban & 12 & 0 \\
\hline Rural & 3 & 0 \\
\hline \multicolumn{3}{|l|}{ Education level } \\
\hline No education & 4 & 1 \\
\hline $1-6$ years & 52 & 0 \\
\hline $7-12$ years & 122 & 4 \\
\hline$>12$ years & 9 & 0 \\
\hline \multicolumn{3}{|l|}{ Socioeconomic level } \\
\hline Low & 37 & 2 \\
\hline Medium & 150 & 3 \\
\hline
\end{tabular}

optical density of the ELISA in the five seropositive cases was $1.35 \pm 0.93$, whereas in the 16 seropositive controls, mean optical density was $0.73 \pm 0.45(\mathrm{P}=0.05)$. Seroprevalence of $E$. histolytica exposure did not vary significantly with age, education, birthplace, residence, or socioeconomic status (Table 1). None of the clinical characteristics of female sex workers including health status, presence or history of lymphadenopathy, frequent abdominal pain, headache or hepatitis were associated with seropositivity to E. histolytica. None of the work characteristics including duration in the occupation, condom use, condom breakage, practice of oral or anal sex, mean number of clients per week, history of injuries during sex work, and history of sexually transmitted diseases were associated with seropositivity to E. histolytica (Table 2). None of the behavioral characteristics of female sex workers including washing hands before eating, consumption of unpasteurized milk or untreated water, consumption of unwashed raw vegetables or fruits, frequency of eating away from home, contact with soil, and foreign travel correlated with seropositivity to E. histolytica.

\section{Discussion}

The association between E. histolytica infection and the oc- cupation of female sex worker has not been studied. Despite $E$. histolytica infection has been linked to sexual activities, there is not currently information about the seroprevalence of E. histolytica infection and risk factors associated with this infection in female sex workers. Therefore, this study aimed to determine whether an association between seropositivity to $E$. histolytica and the occupation of female sex worker exists. For this purpose, we studied the seroprevalence of E. histolytica infection in a sample of registered female sex workers and women without this occupation in the northern Mexican City of Durango. We found that female sex workers had a non-significantly different $(\mathrm{P}=0.48)$ seroprevalence of $E$. histolytica infection than control women without this occupation $(2.7 \%$ vs. $4.3 \%$, respectively). Therefore, our results suggest that $E$. histolytica infection is not associated with the occupation of female sex worker. The low seroprevalence of E. histolytica found in female sex workers is comparable to the E. histolytica seroprevalences $(<5 \%)$ reported in people surveyed in other eight northern Mexican states [21]. However, this comparison should be taken with care since different laboratory methods were used among the studies; in the study of eight Mexican states, a homemade ELISA was used, whereas a commercially available ELISA was used in our study. The seroprevalence of E. histolytica infection found in female sex workers is 
Table 2. Work Characteristics of Female Sex Workers and Seroprevalence of $E$. histolytica Infection

\begin{tabular}{|c|c|c|}
\hline Characteristic & No. of subjects & No. of positive cases \\
\hline \multicolumn{3}{|l|}{ Duration in the activity } \\
\hline Less than 5 years & 16 & 0 \\
\hline $5-10$ years & 45 & 0 \\
\hline More than 10 years & 126 & 5 \\
\hline \multicolumn{3}{|l|}{ Condom use } \\
\hline Yes & 158 & 5 \\
\hline No & 26 & 0 \\
\hline \multicolumn{3}{|l|}{ Condom breakage } \\
\hline Yes & 71 & 1 \\
\hline No & 113 & 4 \\
\hline \multicolumn{3}{|l|}{ Oral sex } \\
\hline Yes & 118 & 3 \\
\hline No & 68 & 2 \\
\hline \multicolumn{3}{|l|}{ Anal sex } \\
\hline Yes & 16 & 0 \\
\hline No & 169 & 5 \\
\hline \multicolumn{3}{|c|}{ Mean number of clients a week } \\
\hline Less than 10 & 124 & 4 \\
\hline $10-20$ & 36 & 0 \\
\hline $21-30$ & 11 & 1 \\
\hline More than 30 & 13 & 0 \\
\hline \multicolumn{3}{|l|}{ Injuries at work } \\
\hline Yes & 11 & 0 \\
\hline No & 173 & 5 \\
\hline \multicolumn{3}{|c|}{ Sexually transmitted diseases } \\
\hline Yes & 47 & 0 \\
\hline No & 140 & 5 \\
\hline
\end{tabular}

slightly lower than the mean $8.41 \%$ seroprevalence found in 32 federal entities and $\leq 8 \%$ in northern states reported in a national study [22]. However, again this comparison should be interpreted with care since different laboratory tests were used among the studies; in the national survey, the presence of antibodies against $E$. histolytica was detected by an indirect hemagglutination test, whereas we used an ELISA. In a second national seroepidemiology survey in Mexico, researchers used an ELISA to detect antibodies against E. histolytica and found a $4.49 \%$ seroprevalence [23], which is comparable to the seroprevalence found in female sex workers. In a local context, the seroprevalence of E. histolytica infection found in female sex workers is much lower than the $41.8 \%$ seroprevalence reported in rural population [24], and the $28.2 \%$ seroprevalence found in the ethnic group of Tepehuanos [20] in Durango State using the same commercially available enzyme immunoassay. The fact that female sex workers had a low seroprevalence of $E$. histolytica compared to those reported in other populations in
Mexico further suggests that female sex workers do not have an increased risk for infection with $E$. histolytica. Interestingly, the mean optical density of the ELISA was higher $(1.35 \pm 0.93)$ in cases than in controls $(0.73 \pm 0.45)$. However, the difference in optical density was of borderline significance $(\mathrm{P}=0.05)$. The lack of association between infection with $E$. histolytica and the occupation of female sex worker was unexpected. Some reports suggest that $E$. histolytica can be sexually transmitted $[8,9]$. An increased seroprevalence of $E$. histolytica infection has been reported in MSM [10-12], especially the ones with HIV infection [13-15]. In addition, heterosexual activity and female homosexual activity have been linked to E. histolytica infection [7]. An increase in the annual rate of anti-E. histolytica seropositivity in female outpatients was observed in a clinic for sexually transmitted diseases in Tokyo [17]. Furthermore, penile amebiasis was reported in men who practiced unprotected anal intercourse [18]. Results of the present study do not exclude a sexual transmission of E. histolytica. The fre- 
quent use of condom among the study population might have influenced the rate of infection.

Potential risk factors associated with E. histolytica in female sex workers were also investigated. In a previous study of general population in rural Durango, we found that seropositivity to E. histolytica was associated with poor education of the head of the family [24]. In a study of northeast Indian population, researchers observed that participants having lower levels of education exhibited a higher infection rate of $E$. histolytica [25]. However, in the present study, no association between $E$. histolytica seropositivity and levels of education of the participants or the head of their families was found. The low seroprevalence of E. histolytica infection found in urban women in the present study was contrast with a higher $(41.8 \%)$ seroprevalence of this infection reported in general population in rural Durango [24]. Poorer sanitation conditions in rural than in urban areas might explain the differences in the seroprevalences.

The present study has some limitations: the number of seropositive subjects was small. In fact, this limitation did not allow us to perform multivariate analysis to determine the association between characteristics of female sex workers and $E$. histolytica infection. We studied only registered female sex workers, and it is unknown whether the lack of association between infection and the occupation of female sex worker found in the present study can also be observed in non-registered female sex workers.

\section{Conclusions}

We conclude that infection with E. histolytica is not associated with the occupation of female sex worker in the northern Mexican city of Durango. Further studies to determine the risk of infection with E. histolytica by sexual contact should be conducted.

\section{Conflicts of Interest}

None.

\section{References}

1. Bercu TE, Petri WA, Behm JW. Amebic colitis: new insights into pathogenesis and treatment. Curr Gastroenterol Rep. 2007;9(5):429-433.

2. Tengku SA, Norhayati M. Public health and clinical importance of amoebiasis in Malaysia: a review. Trop Biomed. 2011;28(2):194-222.

3. Mortimer L, Chadee K. The immunopathogenesis of Entamoeba histolytica. Exp Parasitol. 2010;126(3):366-380.

4. Pritt BS, Clark CG. Amebiasis. Mayo Clin Proc. 2008;83(10):1154-1159; quiz 1159-1160.

5. Chacin-Bonilla L. [An update on amebiasis]. Rev Med Chil. 2013;141(5):609-615.

6. Ralston KS, Petri WA. The ways of a killer: how does Entamoeba histolytica elicit host cell death? Essays Bio- chem. 2011:51:193-210.

7. Salit IE, Khairnar K, Gough K, Pillai DR. A possible cluster of sexually transmitted Entamoeba histolytica: genetic analysis of a highly virulent strain. Clin Infect Dis. 2009;49(3):346-353.

8. Moldwin RM. Sexually transmitted protozoal infections. Trichomonas vaginalis, Entamoeba histolytica, and Giardia lamblia. Urol Clin North Am. 1992;19(1):93-101.

9. Levine GI. Sexually transmitted parasitic diseases. Prim Care. 1991;18(1):101-128.

10. Lee HC, Ko NY, Lee NY, Chang CM, Ko WC. Seroprevalence of viral hepatitis and sexually transmitted disease among adults with recently diagnosed HIV infection in Southern Taiwan, 2000-2005: upsurge in hepatitis C virus infections among injection drug users. J Formos Med Assoc. 2008;107(5):404-411.

11. Zhou F, Li M, Li X, Yang Y, Gao C, Jin Q, Gao L. Seroprevalence of Entamoeba histolytica infection among Chinese men who have sex with men. PLoS Negl Trop Dis. 2013;7(5):e2232.

12. Hung CC, Wu PY, Chang SY, Ji DD, Sun HY, Liu WC, $\mathrm{Wu} \mathrm{CH}$, et al. Amebiasis among persons who sought voluntary counseling and testing for human immunodeficiency virus infection: a case-control study. Am J Trop Med Hyg. 2011;84(1):65-69.

13. Samie A, Barrett LJ, Bessong PO, Ramalivhana JN, Mavhandu LG, Njayou M, Guerrant RL. Seroprevalence of Entamoeba histolytica in the context of HIV and AIDS: the case of Vhembe district, in South Africa's Limpopo province. Ann Trop Med Parasitol. 2010;104(1):55-63.

14. Chen Y, Zhang Y, Yang B, Qi T, Lu H, Cheng X, Tachibana H. Seroprevalence of Entamoeba histolytica infection in HIV-infected patients in China. Am J Trop Med Hyg. 2007;77(5):825-828.

15. Tsai JJ, Sun HY, Ke LY, Tsai KS, Chang SY, Hsieh SM, Hsiao CF, et al. Higher seroprevalence of Entamoeba histolytica infection is associated with human immunodeficiency virus type 1 infection in Taiwan. Am J Trop Med Hyg. 2006;74(6):1016-1019.

16. James R, Barratt J, Marriott D, Harkness J, Stark D. Seroprevalence of Entamoeba histolytica infection among men who have sex with men in Sydney, Australia. Am J Trop Med Hyg. 2010;83(4):914-916.

17. Suzuki J, Kobayashi S, Iku I, Murata R, Yanagawa Y, Takeuchi T. Seroprevalence of Entamoeba histolytica infection in female outpatients at a sexually transmitted disease sentinel clinic in Tokyo, Japan. Jpn J Infect Dis. 2008;61(3):175-178.

18. Abdolrasouli A, de Vries HJ, Hemmati Y, Roushan A, Hart J, Waugh MA. Sexually transmitted penile amoebiasis in Iran: a case series. Sex Transm Infect. 2012;88(8):585588.

19. Gatti S, Cevini C, Bernuzzi AM, Bruno A, Scaglia M. Symptomatic and asymptomatic amoebiasis in two heterosexual couples. Ann Trop Med Parasitol. 1999;93(8):829834.

20. Alvarado-Esquivel C, Hernandez-Tinoco J, Francisco Sanchez-Anguiano L, Ramos-Nevarez A, Margarita Cerrillo-Soto S, Alberto Guido-Arreola C. Serosurvey 
of Entamoeba Histolytica Exposure among Tepehuanos Population in Durango, Mexico. Int $\mathrm{J}$ Biomed Sci. 2015;11(2):61-66.

21. Isibasi A, Gonzalez C, Ortiz V, Muy M, Paniagua J, Blanco F, Pelayo R, et al. [Seroepidemiology of amebiasis in the northern region of the Republic of Mexico]. Arch Invest Med (Mex). 1990;21(Suppl 1):163-174.

22. Caballero-Salcedo A, Viveros-Rogel M, Salvatierra B, Tapia-Conyer R, Sepulveda-Amor J, Gutierrez G, OrtizOrtiz L. Seroepidemiology of amebiasis in Mexico. Am J Trop Med Hyg. 1994;50(4):412-419.

23. Gonzalez CR, Isibasi A, Ortiz-Navarrete V, Paniagua J,
Garcia JA, Ramirez A, Salvatierra B, et al. Prevalence of antibodies against Entamoeba histolytica in Mexico measured by ELISA. Epidemiol Infect. 1995;115(3):535543.

24. Alvarado-Esquivel C, Hernandez-Tinoco J, Sanchez-Anguiano LF. Seroepidemiology of Entamoeba histolytica Infection in General Population in Rural Durango, Mexico. J Clin Med Res. 2015;7(6):435-439.

25. Nath J, Ghosh SK, Singha B, Paul J. Molecular Epidemiology of Amoebiasis: A Cross-Sectional Study among North East Indian Population. PLoS Negl Trop Dis. 2015;9(12):e0004225. 